\title{
Frontières
}

\section{Médiations interdisciplinaires en études sur la mort}

\section{De l'aigu au grave}

\section{Diane Laflamme}

Volume 20, numéro 2, printemps 2008

Les musiques et la mort

URI : https://id.erudit.org/iderudit/018325ar

DOI : https://doi.org/10.7202/018325ar

Aller au sommaire du numéro

Éditeur(s)

Université du Québec à Montréal

ISSN

1180-3479 (imprimé)

1916-0976 (numérique)

Découvrir la revue

Citer ce document

Laflamme, D. (2008). Médiations interdisciplinaires en études sur la mort : de

l'aigu au grave. Frontières, 20(2), 3-6. https://doi.org/10.7202/018325ar d'utilisation que vous pouvez consulter en ligne.

https://apropos.erudit.org/fr/usagers/politique-dutilisation/ 


\section{Médiations interdisciplinaires en études sur la mort De l'aigu au grave}

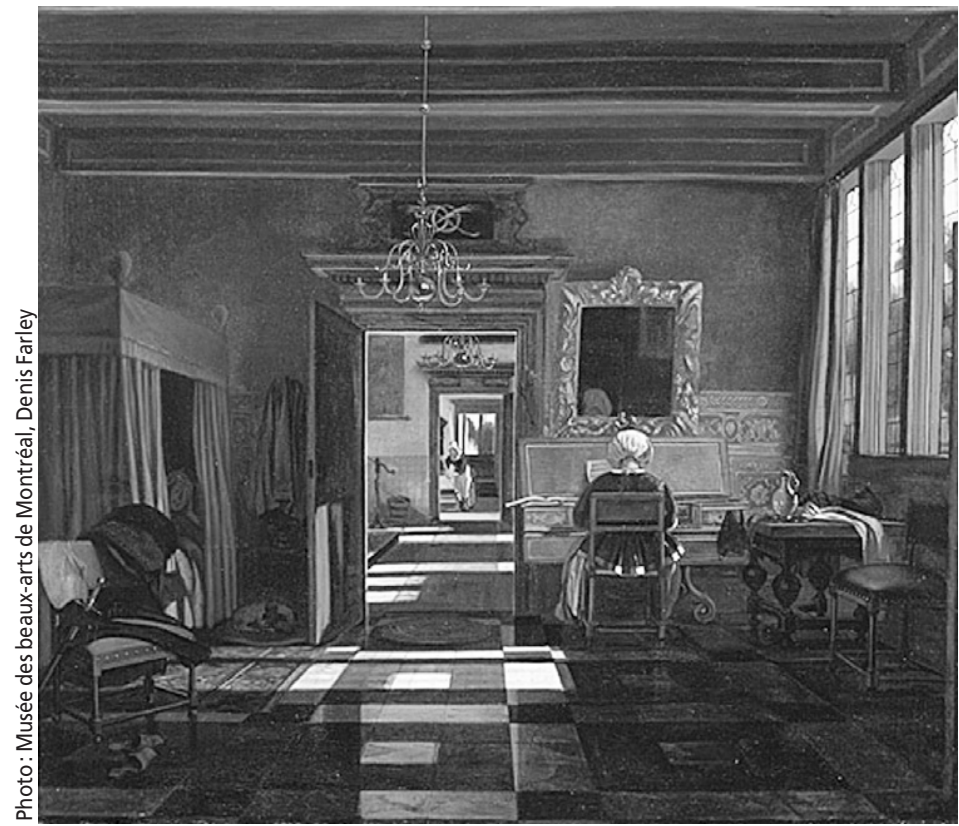

Emanuel De Witte, Intérieur avec une femme jouant de l'épinette, vers 1600 , huile sur toile, $97,5 \mathrm{~cm} \times 109,7 \mathrm{~cm}$, Musée des beaux-arts de Montréal, Fonds John W. Tempest.

\section{Diane Laflamme, Ph. D., rédactrice en chef.}

Est-il devenu présomptueux d'invoquer l'interdisciplinarité dans l'intitulé d'un texte? Tant et tant a été dit et écrit depuis que le mot s'est imposé aux chercheurs, à partir des années 1960, et pourtant «où » est l'interdisciplinarité? À la question «où? » l'interdisciplinarité - comme la musique qu'observe Michel Serres dans l'extrait cité en exergue - ne répond pas, ne répond plus... semble-t-il. À moins que sa réponse ne se déploie sur une longueur d'onde différente de celle vers laquelle nous avons pris l'habitude de tendre l'oreille...

Avouons que la tentation est grande de schématiser à l'emporte-pièce les défis actuels de l'interdisciplinarité. Pour les sciences humaines en général - et pour les études sur la mort en
OÙ SE TROUVE LE REQUIEM DE GABRIEL FAURÉ, OÙ SE TIENT L'CEUVRE DE FRANÇOIS COUPERIN [...] DANS UN ÉCRIT OU UNE PARTITION, MAIS LESQUELS? SUR QUEL INSTRUMENT [...] DANS QUELLE SALLE, SOUS QUELLE BAGUETTE?

ET CEPENDANT LA MUSIQUE OCCUPE L'ESPACE À PARTIR D'UNE ÉCOUTE LOCALISABLE : JUSQU'OÙ? PRÉSENTE LA SONATE ET POURTANT ABSENTE. PARTOUT, PAS TOUT À FAIT PARTOUT, NULLE PART. À LA QUESTION OÙ?, LA MUSIQUE NE RÉPOND PAS.

MICHEL SERRES, STATUES, 1987, P. 333.

particulier -, la situation, brossée à grands traits, se résumerait ainsi : la crise est aiguë et les lacunes sont graves. Dans l'institution universitaire et pour les organismes subventionnaires, les transferts de connaissance propres à alimenter une connaissance interdisciplinaire sont traités en parent pauvre comparativement à la recherche disciplinaire, d'où le climat de crise; trop souvent on se contente de juxtaposer les disciplines, d'où les lacunes.

Pour la revue Frontières, l'interdisciplinarité se vit dans un champ d'application bien précis: les études sur la mort. C'est pourquoi je me propose ici, au lieu de citer les définitions et distinctions ${ }^{1}$ couramment proposées, de voir dans l'interdisciplinarité une "émergence pratique»- émergence comme la musique, et qui plus est, dans le champ des pratiques. Pour ce faire, je me référerai à un article publié en 1999, il y aura bientôt dix ans donc, où Jules Duchastel et Danielle Laberge (1999) 
envisagent l'interdisciplinarité comme: "une émergence pratique d'intersections entre diverses modalités de médiation à l'intérieur même de la recherche».

Depuis sa création, n'est-ce pas là ce que propose aux chercheurs la revue FRONTIÈRES : faire émerger des intersections entre diverses modalités de médiations en les appliquant à des thématiques reliées à la mort et au deuil? Le nombre de disciplines qui ont répondu à cette invitation au cours des vingt années de publication de la revue est impressionnant, même selon un inventaire partiel (voir tableau 1). On pourrait dire, en paraphrasant Michel Serres, que les écrits et les instruments sont là ; mais «où » est l'interdisciplinarité?

Pourquoi chercher ainsi l'émergence de l'interdisciplinarité dans le champ des études sur la mort? Pourquoi l'invoquer, la souhaiter? Parce que l'objet de recherche est ici éminemment plus complexe $^{2}$ que sa représentation disciplinaire. Même en multipliant les angles disciplinaires, ce qu'il y a à connaître au sujet de la fin de la vie nous échappe et nous appelle à poursuivre notre observation, encore et encore, parfois en solo, parfois à l'unisson.

\section{LA REVUE FRONTIÈRES COMME ESPACE DE COCONSTRUCTION INTERDISCIPLINAIRE}

Duchastel et Laberge nous parlent d'un espace de médiation interdisciplinaire, un lieu dans lequel «le chercheur est appelé à interagir avec d'autres disciplines ». Cette interaction se fait, disent-ils, «dans un double mouvement de délimitation (mise en objet propre à sa discipline) et de médiation (coconstruction interdisciplinaire)» (1999, p. 64). Avec ce tempo, nous demeurons en quelque sorte assez proche du vocabulaire de la musique; premier temps du mouvement: la délimitation, qui doit être faite avec toute la compétence et les connaissances spécialisées propres à une discipline; deuxième temps, la médiation proprement dite, c'est-à-dire la coconstruction interdisciplinaire.

En ouvrant ses pages à des auteurs provenant de champs disciplinaires très diversifiés, la revue FRONTIÈRES a-t-elle rendu possible ce double mouvement de délimitation et de médiation? On peut se demander si nos auteurs s'en sont tenus au premier mouvement de cette "valse à deux temps », le travail de délimitation pour lequel leur discipline d'origine les avait très bien pré-

TABLEAU 1

RELEVÉ DES DISCIPLINES MISES À CONTRIBUTION POUR TRAITER DU DEUIL ET DES SOINS PALLIATIFS DANS 6 NUMÉROS DE FRONTIÈRES PUBLIÉS DU PRINTEMPS 2003 À L'AUTOMNE 2005

\begin{tabular}{|c|c|c|c|c|}
\hline \multirow{2}{*}{\multicolumn{2}{|c|}{ Ce qui est observé }} & \multirow{2}{*}{ Par quelle discipline $^{a}$} & \multicolumn{2}{|c|}{6 numéros: du printemps 2003 à l'automne $2005^{b}$} \\
\hline & & & Deuilc $^{c}$ & Soins palliatifs ${ }^{\mathrm{d}}$ \\
\hline \multirow{4}{*}{\multicolumn{2}{|c|}{ La sphère du privée }} & Psychanalyse & - & - \\
\hline & & Psychiatrie & $16-2$ & - \\
\hline & & Psychologie & $16-1 / \mathbf{1 6 - 2}$ & 15-2/16-1/16-2/17-1 \\
\hline & & Psychothérapie & $15-2$ & - \\
\hline \multirow{13}{*}{$\begin{array}{l}\text { L'articulation } \\
\text { du privé } \\
\text { et du public }\end{array}$} & \multirow{6}{*}{$\begin{array}{l}\text { les soins } \\
\text { de santé }\end{array}$} & Médecine & $16-1$ & $16-1 / 17-1$ \\
\hline & & Musicothérapie & $17-2$ & - \\
\hline & & Pharmacie & - & $16-1$ \\
\hline & & Sc. infirmières & $15-2 / 16-2$ & 17-1 \\
\hline & & Sexologie & $16-1 / 16-2$ & $16-1 / \mathbf{1 7 - 1}$ \\
\hline & & Travail social & $16-1 / 16-2$ & $16-1 / \mathbf{1 7 - 1}$ \\
\hline & l'économie & - & - & - \\
\hline & \multirow{4}{*}{ la culture } & Anthropologie & $16-1$ & $16-1 / 16-2$ \\
\hline & & Études littéraires & $15-2 / 16-2$ & $17-1$ \\
\hline & & Sc. de l'éducation & - & $17-1$ \\
\hline & & Sc. des religions & $15-2 / 18-1 / \mathbf{1 6 - 2}$ & $17-1$ \\
\hline & \multirow{2}{*}{ le politique } & Sc. juridiques & $15-2$ & $17-1$ \\
\hline & & Sc. politiques & - & 17-1 \\
\hline \multirow{4}{*}{\multicolumn{2}{|c|}{ L'entièreté de l'expérience sociale }} & Éthique & - & $17-1$ \\
\hline & & Histoire & - & - \\
\hline & & Philosophie & $16-1$ & $17-2$ \\
\hline & & Sociologie & $16-1$ & - \\
\hline
\end{tabular}

a. La discipline mentionnée correspond au champ de rattachement disciplinaire indiqué par l'auteur du texte pour s'identifier auprès de la revue.

b. Les numéros choisis sont ceux qui encadrent (2 numéros avant et 2 numéros après) les deux numéros se rattachant plus précisément au deuil (16-2) et aux soins palliatifs (17-1) Ces deux numéros pivots sont inscrits en gras dans le tableau. Les six numéros recensés portaient sur les thématiques suivantes : 15-2, Guerre, mort amère; 16-1, Remède ou poison?; 16-2, Deuil, blessure vive; 17-1, Au péril de l'accompagnement; 17-2, Surtout, ne pas souffrir; 18-1, Hélas, célébrer la mort!

c. Une entrée a été effectuée lorsque le numéro comportait un texte où figurait le mot «deuil». Les textes recensés faisaient partie des sections: Articles, Recherche, Intervention, Points de vue ou Regards. Une entrée peut faire référence à plus d'un texte.

d. Une entrée a été effectuée lorsque le numéro comportait un texte où figuraient les mots «soins palliatifs» ou «accompagnement» des mourants. Les textes recensés faisaient partie des sections: Articles, Recherche, Intervention, Points de vue ou Regards. Une entrée peut faire référence à plus d'un texte.

e. Le regroupement des disciplines proposé dans ce tableau s'inspire de Duchastel et Laberge (1999, p. 69-70) avec des ajouts pour tenir compte de toutes les contributions disciplinaires accueillies par la revue FRONTIĖRES au cours de la période considérée. 
parés, ou s'ils ont su tirer profit de l'espace que leur proposait la revue pour tenter et réussir des médiations interdisciplinaires.

Duchastel et Laberge (1999, p. 64-68) distinguent quatre dimensions de médiation: la construction de l'objet d'étude, les engagements épistémologiques, les déterminations méthodologiques et l'interprétation globale de l'objet. Voici quelques exemples de la réflexion qu'un tel découpage pourrait susciter dans le champ des études sur la mort.

- La construction de l'objet d'études: En études sur la mort, nous travaillons avec plusieurs définitions de la perte, du deuil, des soins palliatifs, du suicide, de la souffrance, etc.; ces définitions que chaque chercheur emprunte à sa discipline d'origine, comment se sont-elles enrichies au contact des autres disciplines? La spécification disciplinaire de l'objet de recherche a-t-elle servi de point de départ pour une démarche concertée visant à élargir la compréhension du phénomène à l'étude? Après avoir rassemblé autour d'une même problématique les contributions de chacune des disciplines, laisse-t-on le plus souvent à la lectrice ou au lecteur la tâche de produire la réflexion interdisciplinaire?

- Les engagements épistémologiques : La recherche sur les phénomènes observés en relation avec la fin de la vie fournit-elle au chercheur une occasion de se confronter à d'autres positions que la sienne sur des questions comme la nature du réel et le statut de vérité de la connaissance? Assiste-t-on à des rapprochements entre chercheurs qui s'inspirent d'un même paradigme, alors que leur discipline d'origine tendrait plutôt à les entraîner sur des voies divergentes?

- Les déterminations méthodologiques: Le regroupement en équipes pluridisciplinaires de chercheurs et d'intervenants provenant des milieux de pratique suscite-t-il une mise en commun des méthodes de travail, un échange entre des savoir-faire utiles entre autres pour la saisie, la description, la catégorisation, la comparaison et l'analyse des matériaux de recherche? Assiste-t-on à un apprivoisement réciproque entre les membres de l'équipe en ce qui a trait aux opérations et aux habiletés qui permettent tant au chercheur qu'au praticien de circuler de l'abstrait au concret et du concret à l'abstrait?

- L'interprétation globale de l'objet: Sur diverses thématiques relatives à la mort et au deuil, peut-on répertorier des recherches qui examinent un ensemble d'hypothèses interprétatives afin de rendre compte de la complexité du phénomène à l'étude en fonction de plusieurs disciplines? Quelle est la contribution des recherches qui maintiennent une spécialisation disciplinaire tout en faisant référence à des ressources interdisciplinaires gardées prudemment à l'arrière-plan?

Un examen des articles publiés dans les dossiers thématiques proposés par FRONTIËRES au cours des dernières années pourrait utilement documenter comment ces quatre médiations interdisciplinaires ont été déployées et jusqu'à quel point elles ont contribué à une meilleure intelligibilité des thèmes abordés.

Il faut souhaiter que des chercheurs s'intéressent à une telle observation, qui ne nous renseignerait pas seulement sur le développement des études sur la mort comme science humaine appliquée, mais également sur le développement de chacune des disciplines qui a été mise à contribution dans cet espace de médiation et qui devrait, dès lors, être sortie de cet exercice elle-même transformée. L'interdisciplinarité est émergence, nous l'avons déjà dit; ce qu'elle fait émerger, c'est la présence de l'autre au sein du même. En même temps qu'elle participe à une coconstruction interdisciplinaire dans un champ d'application donné, la discipline elle-même se co-construit. Nous assistons à «la construction d'une discipline dans un espace permanent de médiation », déclare Berthelot dans son commentaire de la proposition de Duchastel et Laberge (1999, p. 5).
En définitive, c'est la conscience réflexive qui est confrontée à la complexité du monde ${ }^{3}$ qu'elle habite et qui est sollicitée pour examiner les conditions de validité de ses propres efforts visant à rendre compte des phénomènes qu'elle observe. C'est la qualité du regard du chercheur qui compte; dans un article portant plus précisément sur la notion de complexité, Jacques Ardoino le rappelle clairement:

Il n'y a sans doute pas réellement dans l'univers, de façon absolue, une dichotomie possible entre des objets simples, d'une part, et des objets complexes, d'autre part. Il y a plutôt, nous semble-t-il, des données et les idées que nous élaborons à leur propos. C'est donc sur la qualité du regard du chercheur, quand les entreprises d'intelligibilité plus classiques, canoniques, se seront avérées vaines, qu'il convient surtout de réfléchir, plutôt que sur les propriétés ainsi prêtées aux matériaux de la recherche (1999, p. 444).

Le chercheur voudra tantôt inscrire sa production scientifique dans un encadrement disciplinaire strict, tantôt explorer des médiations interdisciplinaires susceptibles de contribuer autrement à l'intelligibilité des phénomènes étudiés. Quel que soit le champ disciplinaire qui lui sert de tremplin pour se déployer, c'est à la pensée réflexive que revient la tâche de faire advenir l'interdisciplinarité, d'actualiser toutes ces médiations interdisciplinaires qui sont de l'ordre du possible mais qui ne s'imposent pas d'elles-mêmes dans la démarche du chercheur. Loin d'être un incontournable, l'interdisciplinarité est une musique qui surgit suite à l'effort et à la virtuosité d'un interprète.

Le travail de coconstruction interdisciplinaire mobilise la conscience qui, pour reprendre le vocabulaire de la phénoménologie husserlienne, «reçoit le monde», alors que ce monde «se donne» à elle dans toute sa complexité ${ }^{4}$. Edgar Morin attire l'attention sur des aspects précis de ce travail lorsqu'il met en contraste ce qu'il appelle la pensée simplifiante et la pensée complexe:

Ainsi, par exemple, si nous essayons de penser le fait que nous sommes des êtres à la fois physiques, biologiques, sociaux, culturels, psychiques et spirituels, il est évident que la complexité [la pensée complexe] est ce qui essaie de concevoir l'articulation, l'identité et la différence de tous ces aspects, alors que la pensée simplifiante soit disjoint ces différents aspects, soit les unifie par une réduction mutilante. Donc, en ce sens-là, il est évident que l'ambition de la complexité [de la pensée complexe] est de rendre compte des articulations qui sont brisées par les coupures entre disciplines, entre catégories cognitives et entre types de connaissances (Morin, 1984, p. 80).

Au-delà des textes et des instruments, « où » se trouve l'interdisciplinarité, demandions-nous? Elle habite le travail de la pensée complexe, c'est là son espace d'émergence. L'interdisciplinarité cherche les articulations, les identités, les différences; elle effectue des "transports", au sens où l'entend Michel Serres dans sa théorie des transports et messageries reliant les unes avec les autres tant les disciplines scientifiques que la philosophie, les mathématiques, l'art et la littérature (1969, 1972, 1974). L'interdisciplinarité fait œuvre de transfert de connaissance (Hamel, 1997, p. 250) non pas "à côté» de la recherche, mais en suscitant des médiations à l'intérieur même du processus de recherche.

Avec le mot médiations, le titre de ce texte nous invite à explorer des positions plus nuancées que le diagnostic trop simple que serait le constat des crises aiguës et des lacunes graves invoquées au deuxième paragraphe. Il importe de tenir compte des avancées bien réelles et des succès même partiels de l'interdisciplinarité dans divers champs d'application, notamment les études sur la mort. Ces avancées et ces progrès, il faut les dire, les décrire et s'en servir pour aller plus loin. Le chemin de l'interdisciplinarité 
est déjà balisé, il faut le poursuivre avec une conscience aiguë des enjeux et, oserons-nous le dire, une allégresse grave. Allégresse, parce que toute œuvre de connaissance donne des ailes; grave, parce que ces ailes nous portent vers la haute mer.

\section{Bibliographie}

ARDOINO, J. (1999). «La complexité», dans E. MORIN (dir.), Le défi du XXI ${ }^{e}$ siècle. Relier les connaissances, Paris, Seuil, p. 442 à 450.

BERTHELOT, JEAN-MICHEL (1999). «Présentation», dans Sociologie et sociétés, vol. XXXI, $\mathrm{n}^{\circ}$ 1, printemps, p. 3-10.

DUCHASTEL, J. et D. LABERGE (1999). "La recherche comme espace de médiation interdisciplinaire », dans Sociologie et sociétés, vol. XXXI, n 1, printemps, p. 63-76.

HAMEL, J. (1997). Précis d'épistémologie de la sociologie, Montréal, Paris, L'Harmattan.

LUHMANN, N. (1995). Social Systems, Stanford, Californie, Stanford University Press.

MATHURIN, C. (2002). "Aspects de l'interdisciplinarité: Essai de reconstitution d'un débat», dans L. GÉLINEAU (dir.), L'interdisciplinarité et la recherche sociale appliquée, Université de Montréal, disponible en ligne, $<\mathrm{http}: / / \mathrm{ww}$ w.fes.umontreal.ca/sha/ L'interdisciplinarite>

MORIN, E. (1984). «Sur la définition de la complexité », dans Science et pratique de la complexité, Actes du Colloque de Montpellier, Paris, La Documentation française et IDATE/ Université des Nations Unies, p. 79-85.

MUCCHIELLI, A. (1996). "Complexité (paradigme de la)», dans A. MUCCHIELLI (dir.), Dictionnaire des méthodes qualitatives en sciences humaines et sociales, Paris, Armand Colin, p. 29.

SERRES, M. (1987). Statues, Paris, Éditions François Bourin. SERRES, M. (1969, 1972, 1974). Hermès, I. La communication, II. L'interférence, III. La traduction, Paris, Éditions de Minuit.

\section{Notes}

1. Pour une présentation approfondie de la notion d'interdisciplinarité, voir Creutzer Mathurin (2002). Citons également la présentation concise qu'en fait Berthelot dans le texte d'ouverture du numéro de Sociologie et sociétés consacré à l'interdisciplinarité: "On retiendra, pour fixer le vocabulaire, que l'interdisciplinarité implique, à la différence de la juxtaposition à laquelle se réduit souvent la pluridisciplinarité, un effort d'intégration et de traduction au moins partielles, alors que la transdisciplinarité désigne des concepts, des thèmes ou des schèmes communs à diverses disciplines » (1999, p. 4).

2. Le paradigme de la complexité est défini comme suit par A. Mucchielli (1996) dans son Dictionnaire des méthodes qualitatives en sciences humaines et sociales (p. 29): "Cadre épistémologique, théorique et conceptuel servant à l'étude des phénomènes humains et sociaux. Les théories, les concepts et les situations référentielles utilisées dans ce paradigme sont issus de la systémique et du constructivisme. Par ailleurs, l'attitude épistémologique de ce paradigme repose sur la volonté de produire des connaissances spécifiques aux sciences humaines et sociales à travers une attention particulière portée aux acteurs et aux significations. Il met aussi en avant trois principes: le principe dialogique (le dépassement des antagonismes dans une construction supérieure), le principe de récursion (les effets circulaires et en boucle qui affectent tout phénomène humain), le principe hologrammatique (qui met en évidence que le tout est dans la partie comme celle-ci se retrouve dans le tout).»

3. Au sujet du monde, envisagé comme simple ou complexe, Jacques Ardoino écrit: «Le monde, considéré sous l'angle de ses régularités, comme obéissant à un principe d'ordre et d'organisation, est simple, tant qu'on le voit tel. Il se complexifie à partir du moment où une intelligence du désordre s'élabore pour affiner, étoffer et rendre plus subtil le regard porté sur les phénomènes. Ceux-ci ne sont plus alors supposés divisibles, susceptibles d'être réduits en éléments plus simples, au gré d'une analyse-décomposition, mais ils sont, tout au contraire, postulés indécomposables, de par la nature même du tissage qui les constitue, en fonction de l'intelligence qui veut les appréhender. L'ensemble interactif (système singulier fonctionnant comme un réseau) laissera entrevoir, plus profondément, des hiérarchies enchevêtrées à partir desquelles on se représentera des effets contrariants, voire antagonistes, n'interdisant pourtant pas le maintien d'une cohérence du discours scientifique» (1999, p. 444)

4. Je me réfère ici à la définition de la complexité que propose le sociologue Niklas Luhmann à partir des deux notions d'éléments et de relations entre les éléments. Le regroupement de plusieurs éléments qui se trouvent en interrelation est dit complexe lorsque, compte tenu des contraintes immanentes qui s'exercent sur la capacité de connexion de ces éléments, il devient impossible de relier, chacun à chacun, tous les éléments.

Cela s'applique entre autres aux regroupements que Luhmann appelle les systèmes producteurs de sens, c'est-à-dire les systèmes psychiques, qui produisent du sens en produisant des pensées qui se connectent l'une à l'autre, et les systèmes sociaux, qui produisent du sens en produisant des communications qui se connectent aussi l'une à l'autre.

Comme il y a dans le monde plus de possibilités de connexions que dans les systèmes qui s'y trouvent, on le dit plus complexe que ces derniers. Pour les systèmes qui produisent du sens, le monde fonctionne comme l'horizon ultime des opérations productrices de sens, comme l'a déjà décrit la phénoménologie husserlienne. En tant que réservoir de sens, le monde est accessible à partir de n'importe quelle situation: à partir de n'importe quelle pensée ou communication qui sert de point de départ, le système peut avoir accès aux autres possibilités de sens qu'offre le monde en sélectionnant une connexion plutôt qu'une autre entre ses pensées, s'il s'agit d'un système psychique ou entre ses communications, s'il s'agit d'un système social. Par ailleurs, le monde n'est pas pour autant une simple somme de toutes les opérations productrices de sens qui s'y actualisent. Pour les systèmes psychiques et pour les systèmes sociaux, le monde demeure un horizon irréductible (voir Luhmann [1995], Introduction et chapitres 1 et 2). 